\title{
Muon detection at FCC-ee
}

\author{
Sylvie Braibant ${ }^{1,2}$, Paolo Giacomelli ${ }^{1, a}$ \\ ${ }^{1}$ INFN, Sezione di Bologna, Viale Berti Pichat 6/2, 40127 Bologna, Italy \\ 2 Dipartimento di Fisica e Astronomia, University of Bologna, Viale Berti Pichat 6/2, 40127 Bologna, Italy
}

Received: 5 May 2021 / Accepted: 23 October 2021

(C) The Author(s) 2021, corrected publication 2022

\begin{abstract}
Muons provide a clean experimental signature, typically traversing the whole experimental apparatus without decaying. Muon detection systems are therefore usually located at a rather large distance from the primary interaction vertex after all other subdetectors. As such, experimental apparatuses at FCC-ee will certainly employ very large muon systems, covering areas of a few thousand square meters. For obvious reasons of cost, the most suitable detectors to realise these large muon systems are gas detectors. In particular, in recent years, micro-pattern gas detectors (MPGDs) have undergone very interesting developments, providing several new types of detectors with very good spatial and time resolution, high efficiency, high rate capability and high radiation tolerance. The good position and time resolution makes a MPGD an excellent particle tracker, reconstructing tracks at 4-5 m from the primary interaction vertex with sub-mm precision. Therefore MPGDs, apart from efficiently detecting muons, can precisely track and help identifying also hypothesized long lived particles (LLP) that would decay outside of the central trackers. MPGDs have the distinct advantage of being, at least for some detectors and some parts of them, mass-producible by industry, since they employ materials and manufacturing procedures that are used extensively for printed circuit boards (PCB) production. A particularly innovative MPGD, the $\mu$ RWELL, is considered as a possible candidate to build the large muon system of the IDEA detector concept for FCC-ee and is described in some more detail. Other technologies that could be considered for the realisation of muon detection systems are also briefly discussed.
\end{abstract}

\section{Introduction}

In experiments at colliders, the muon plays a special role as a relatively heavy long-lived particle with only electromagnetic interactions. Therefore, a muon typically traverses the whole experimental apparatus before decaying and can be easily identified. Moreover, muons in the final state provide a characteristic clean experimental signature for many theoretical models. For this reason, muons could also provide clear signatures for new physics beyond the Standard Model that could be searched for at FCC-ee [1]. A muon spectrometer [2] consists of a position-sensitive detector that reconstructs the tracks of charged particles and a magnetic field so that charge and momentum can be deduced from the track curvature.

Most recent and current large experimental apparatuses at high-energy colliders have a cylindrical structure closed at the two ends by two endcaps. The central tracker is typically the

\footnotetext{
a e-mail: paolo.giacomelli@bo.infn.it (corresponding author)
} 
sub-detector closest to the interaction point (IP), followed at increasing radial distance from the IP by the electromagnetic and hadron calorimeters. These sub-detectors are surrounded by a solenoid that provides the magnetic field. Given the muon characteristics, the muon detection system is almost always the last sub-detector in the apparatus, located at several meters of distance from the primary vertex, often composed of several stations interleaved in the iron of the magnetic field return yoke. It is foreseeable that future detectors at future accelerators will adopt a similar design. In this paper, we will be using the FCC-ee as a benchmark. All the detectors at FCC-ee will have a length exceeding $12 \mathrm{~m}$ and a radius of at least 5-6 m. Muon detection systems being the outermost detectors will therefore need to cover an overall surface of a few thousands of square meters. For this reason, the technology of choice, apart from having an extremely high efficiency $(\geq 98 \%)$ for muons over the whole surface, needs to have a reasonable price/surface cost and a relatively limited number of channels, such that the readout electronics does not inflate the overall cost of the muon detector.

FCC-ee will operate at four different centre-of-mass energies, ranging from the $\mathrm{Z}$ peak to the $t \bar{t}$ threshold. At these energies, the highest muons momenta typically do not exceed 80$90 \mathrm{GeV}$. The highest muon rates, of the order of a few $\mathrm{kHz}$, will be obtained while running at the $\mathrm{Z}$ peak where the FCC-ee will reach its maximum instantaneous luminosity. These rates, especially when considering the large size of the muon detection systems, mean that the average occupancy of the detectors will be very low.

\section{Muon detection}

Muon detection systems have to identify muons with a very high efficiency. Muon systems are typically composed of several stations of tracking detectors located at several meters of distance from the primary interaction point. For a muon detector at a leptonic collider like FCC-ee, it is possible to envisage two possible working scenarios: (a) a simple muon tagger that would measure with a precision of $>2-3 \mathrm{~mm}$ the position of the hits left in the muon detector and confirm that a charged particle track reconstructed in the central tracker of the experiment is indeed a muon, or (b) a more sophisticated muon detector that measures the position of the hits in the detector with high precision, $\mathrm{O}(100 \mu \mathrm{m})$, and provides an accurate standalone momentum measurement that can be used to match the track reconstructed by the central tracker and complement its measurement. In some cases, one may also require the muon detector to provide a standalone muon trigger and bunch-crossing identification, even though this feature is less relevant for a lepton collider than it is for a hadron collider where the background and particle rates are much higher.

As mentioned above, muon detectors are typically placed after all other sub-detectors, and especially behind the calorimeters that absorb all the electromagnetic and hadronic showers produced. Therefore, the particle rates the muon detector are subject to are typically much lower than for the other sub-detectors. Future leptonic circular colliders like FCCee and CEPC will however be able to run at extremely high instantaneous luminosities, especially at the lower centre-of-mass energy corresponding to the $\mathrm{Z}$ peak. For example, FCC-ee should deliver its highest luminosity $L \approx 2.5 \times 10^{36} \mathrm{~cm}^{-2} \mathrm{~s}^{-1}$ when operating at the $\mathrm{Z}$ peak. Considering the $\mathrm{e}^{+} \mathrm{e}^{-} \rightarrow \mathrm{Z}$ production cross section, this corresponds to a rate of about $100 \mathrm{kHz}$ of $\mathrm{Z}$ boson events. Taking into account the branching ratio of $\mathrm{Z} \rightarrow \mu^{+} \mu^{-}$ decays of $\sim 3.3 \%$, this would translate to $3.3 \mathrm{kHz}$ of di-muon events in the detector. Given the very large size of the detector, the individual channel occupancy would be rather low. Using as an example the proposed IDEA muon detector, the data size produced, considering 
also an estimate of the electronic noise, would be about 2.5 MBytes/s, a manageable data rate.

\section{Detector technology options}

There are several detector technologies that could be employed to build a muon detection system. Solutions that could be used for an experiment at FCC-ee range from scintillator strips or tiles, to a large variety of different types of gas detectors. Scintillators have become even more interesting since the advent of silicon photomultipliers (SiPM) [3,4], to collect and amplify the scintillation light. In particular, long bars $(4-5 \mathrm{~m})$ of thin $(1-2 \mathrm{~cm})$ plastic scintillators, read at the two ends by SiPMs, could allow the realisation of a muon detection system at a relatively moderate cost. Plastic scintillators and SiPMs are easy to operate and would produce a fast electrical signal, with a manageable background noise. Scintillators, especially if used in a proposed triangular shape bars, could achieve a spatial resolution of a few $\mathrm{mm}$. Essentially, most of the recent large experiments [5-8] have been using gas detectors for their muon systems. Commonly used technologies are multiwire proportional chambers (MWPCs) [9,10], drift tubes [10], among which the monitored drift tubes (MDT) [11,12] have been used to realise the very large ATLAS muon detection system [5], limited streamer "Iarocci" tubes [13], resistive plate counters (RPCs) [14,15], and many others. A schematic drawing of an RPC module is shown in Fig. 1.

Starting from the basic RPC design, many variants of RPCs have been built and operated in several high-energy physics experiments. For example, different resistive materials have been used and schemes with several gas gaps have been developed leading to extremely fast $(<1 \mathrm{~ns})$ signals. The dimensions of the readout strips or pads have also been varied extensively but typically large-size RPCs reconstruct the position of a traversing particle with a precision of a few $\mathrm{mm}$. Given these characteristics and their rather high detection efficiency for charged particles, RPCs are an excellent detector choice to realise a muon tagger for an experiment at FCC-ee. The CLD detector concept [16] devised for FCC-ee envisages using 6-7 layers of RPC detectors for realizing its muon identification system. The layers would be installed in the iron return yoke. Each layer would have a modular design with RPC cells of $30 \times 30 \mathrm{~cm}^{2}$. CLD also considers as an alternative possibility to use scintillator bars placed in two perpendicular directions in each muon station. The drawback of RPCs and scintillators is that their position resolution would probably not be sufficient to perform accurate standalone track reconstruction and would not help in identifying LLPs.

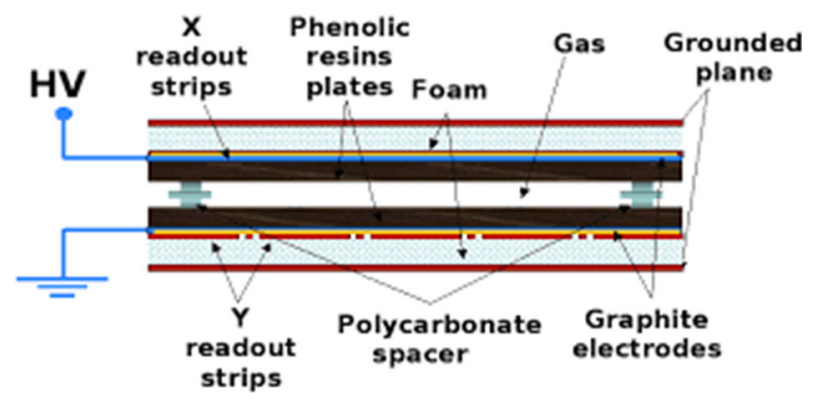

Fig. 1 Schematic drawing of a resistive plate counter 
In recent years, a newer type of gas detectors, the micro-pattern gas detectors (MPGDs) [17] have attracted a lot of attention. MPGDs were developed to overcome some of the limitations of MWPCs, like the slow ion motion and the limited multi-track separation. The aim was therefore to reduce the size of the region where the gas amplification occurs, to achieve a faster ion evacuation and a higher spatial resolution. MPGDs employ the same working principle of MWPCs, with a conversion region, characterized by a low E field, and separate well-localised regions with a high E field, where the multiplication happens. Differently from the classical gas counters, where the distances between anodic and cathodic electrodes are of the order of several mm, in MPGDs, the ionisation charge produced by the charged particle in the gas is multiplied by microstructures with distances between electrodes of the order $10-100 \mu \mathrm{m}$, resulting in a much faster time response. These architectures are implemented for example by micro-holes in GEM [18] and $\mu$ RWELL [19-21] and by micro-mesh in MicroMegas [22]. The last generation of MPGDs exploits modern photolithography technology on flexible and rigid supports. As an example, GEM and MicroMegas are successfully operated in COMPASS [23-25], LHCb and TOTEM [26] experiments at CERN, in KLOE [27] at Frascati-INFN, and are being built for the upgrades of the ATLAS [28,29] and CMS $[30,31]$ experiments at LHC.

The $\mu$ RWELL inherits the best characteristics of the GEM and MicroMegas detectors, while further simplifying the manufacturing process. A technology transfer with a few industries is already in place, and this should allow to be able to mass manufacture this detector in the near future at a very competitive cost of about 1000 euros $/ \mathrm{m}^{2}$. This detector is therefore an ideal candidate to be the technology of choice for building future large muon detection systems [32]. The $\mu$ RWELL technology is in fact envisaged to realise the muon detection system of the IDEA detector concept $[1,33]$ that is proposed for the FCC-ee and CEPC [33] future large circular leptonic colliders. A $\mu$ RWELL detector is composed of two PCBs: a standard GEM Drift PCB acting as the cathode and a $\mu$ RWELL PCB that couples in a unique structure the electron amplification (a well-patterned matrix) and the readout stages, as shown in Fig. 2a). A standard $50 \mu \mathrm{m}$ GEM polyimide foil is copper clad on the top side and diamond-like carbon (DLC) sputtered on the opposite side. The thickness of the DLC layer is adjusted according to the desired surface resistivity value (50-200 $\mathrm{M} \Omega / \square$ ) and represents the bottom of the well matrix providing discharge suppression as well as current evacuation. The foil is then coupled to a readout board (Fig. 2b). A chemical etching process is then performed on the top surface of the overall structure in order to create the WELL pattern (truncated cone wells of $70 \mu \mathrm{m}(50 \mu \mathrm{m})$ top (bottom) in diameter and $140 \mu \mathrm{m}$ pitch) that constitutes the amplification stage (Fig. 2c). The high voltage applied between the copper and the resistive DLC layers produces the required electric field within the WELLs that is necessary to develop charge amplification. The signal is capacitively collected at the strips/pads on the readout boards. In recent years many $\mu$ RWELL detector prototypes have been built and operated in test beams $[20,21]$. Typically a $\mu$ RWELL detector is operated at a high voltage providing a gas gain of a few thousand. In these conditions, a space resolution down to $60 \mu \mathrm{m}$ has been obtained and a time resolution of about $5 \mathrm{~ns}$, with a particle rate capability exceeding a few tens of $\mathrm{kHz} / \mathrm{cm}^{2}$ [34]. For the IDEA $\mu$ RWELL detectors, the signals will be collected in two consecutive layers of strips placed in a perpendicular direction to each other, in order to provide a precise determination of the $\mathrm{x}$ and $\mathrm{y}$ coordinates. A distinctive advantage of the proposed $\mu$ RWELL technology is that the detector does not require complex and time-consuming assembly procedures (neither stretching nor glueing). Being composed of only two main components, the cathode and anode PCBs, the final detector assembly is extremely simplified. 

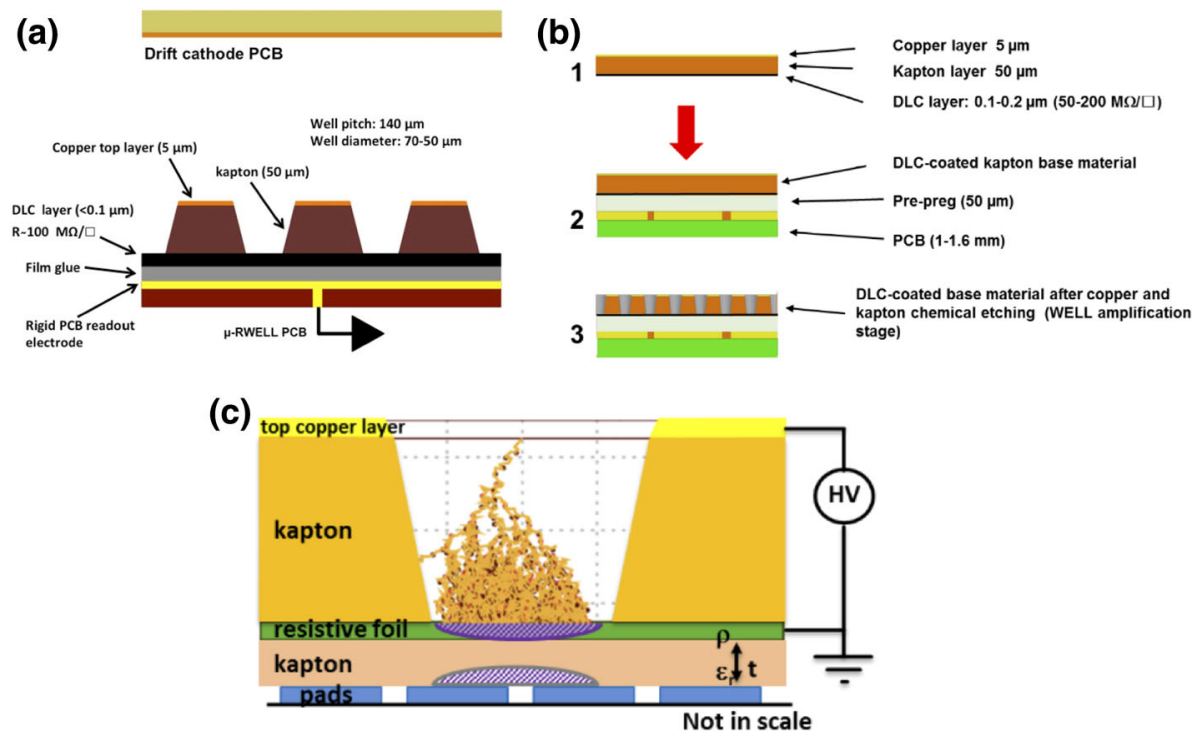

Fig. 2 a Layout of a $\mu$ RWELL detector module; b Coupling steps of the $\mu$ RWELL PCB; c Amplification stage directly coupled with the readout

Both RPCs as well as MPGDs could be mass-produced by industry as their materials and construction procedures have been or could be transferred to industry.

Muons can also be identified by the recent high-definition 3D-imaging calorimeters that are envisaged for FCC-ee. These sophisticated multi-channel calorimeters can in fact accurately reconstruct the charged particles that reach the last layers in the calorimeter, helping to identify them as muons. These high-resolution calorimeters can also provide timing information, effectively providing a complete 4D information on the various particles that are generated in a shower. This information can allow muon identification, which can then be used in conjunction with the muon system downstream.

\section{A conceptual implementation: the IDEA muon detector}

The IDEA detector concept [35] foresees a muon detection system that would be realised using the $\mu$ RWELL technology previously described. The muon detector would follow the IDEA geometry with a central cylindrical barrel region closed at the two extremities by two endcaps to ensure hermeticity. In the barrel, there will be three muon stations, at increasing radial distance from the interaction point, housed within the iron yoke that closes the solenoidal magnetic field. Each station will consist of a large mosaic of $\mu$ RWELL detectors. In order to profit of the industrial production capabilities of this technology, a modular design has been adopted. The basic $\mu$ RWELL "tile" will have an active area of $50 \times 50 \mathrm{~cm}^{2}$. The two layers of strips will both have a strip pitch of $1 \mathrm{~mm}$, giving a total of $500 \times 2$ strips and consequently 1000 readout channels per tile. The detector dimensions, the strip pitch and width are a compromise between the largest $\mu$ RWELL detector that can be industrially mass produced while maintaining a not too high input capacitance to the readout electronics. Table 1 lists the dimensions, number of basic $\mu$ RWELL tiles and readout channels of the 
three muon stations. The two endcaps are made of three disks, at increasing distance from the interaction point in the direction along the beam line equipped with similar $\mu$ RWELL tiles. In total, between the barrel and endcap muon stations, there would be about $5800 \mu$ RWELL tiles with a total of roughly 6 million readout channels. A schematic drawing of a barrel station of the muon detector is shown in Fig. 3. Each $\mu$ RWELL would be able to identify muon hits with 98-99\% efficiency and measure the coordinate perpendicular to the strip direction with a precision of about $200 \mu \mathrm{m}$. Such a detector would be able to provide three three-dimensional space points (the third coordinate coming from the known radial position of the $\mu$ RWell tile) and from these reconstruct the tracks crossing the muon stations. These muon tracks could then be used to complement the tracks reconstructed in the central tracker providing the best momentum measurement of muons. The muon detector could also reconstruct charged particle tracks coming from the decays of hypothesized long-lived particles (LLP) that would produce a secondary vertex outside ( $\geq 2.5 \mathrm{~m}$ from the primary interaction point) of the central tracker. This could significantly enhance the detector capabilities to study these interesting signatures of possible new physics beyond the standard model.

Since the $\mu$ RWELL technology has not yet been used to realise a full detector system a vigorous $R \& D$ program to study integration issues will be carried out in the coming years. The detailed layout of the muon detector, together with all its services, will have to be accurately developed and optimised. The optimal characteristics of the basic $\mu$ RWELL tile, like gas gap, DLC resistivity, strip pitch, and gas amplification will be finalised to match the requirements of the IDEA muon detection system. Another important aspect of the R\&D program will be the design and development of a dedicated front-end electronics based on a custom-made ASIC for the muon detector readout. With this development, the overall cost of the IDEA muon detector could probably not significantly exceed the cost of a simpler muon tagger that, on the other hand, would not have the same capabilities for studying LLPs. Figure 4 shows an event display of a simulated Higgstrahlung event where a Higgs boson is produced in association with a $\mathrm{Z}$ boson at a centre-of-mass energy of $240 \mathrm{GeV}$. The $\mathrm{Z}$ boson then decays to a muon pair.

\section{Conclusions}

Muon detectors play an important role in all modern experimental apparatuses at colliders and will continue to do so also at FCC-ee. They are typically composed of tracking chambers placed at several meters of distance from the primary interaction vertex. Due to the clean environment of FCC-ee, muon rates will be at most of the order of a few $\mathrm{kHz}$ and the highest momenta muons produced will be below $100 \mathrm{GeV}$. Several detector technologies have been used to realise muon detectors for recent collider experiments. Gas detectors are arguably the most suited technology for muon detection at FCC-ee. In particular, micro-pattern gas detectors offer interesting characteristics and could be mass produced by industry. The proposed muon detection system of the IDEA detector concept, realised using $\mu$ RWELL technology, is a possible innovative solution with very good performance in terms of efficiency, space and time resolution. 


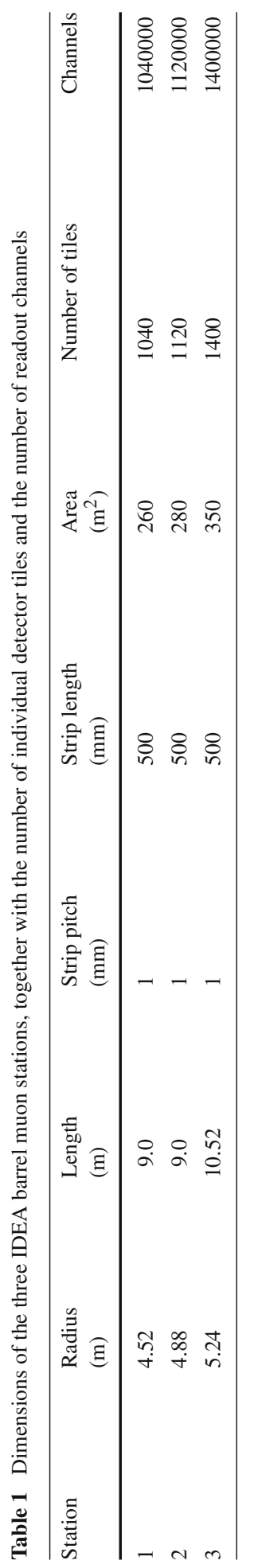




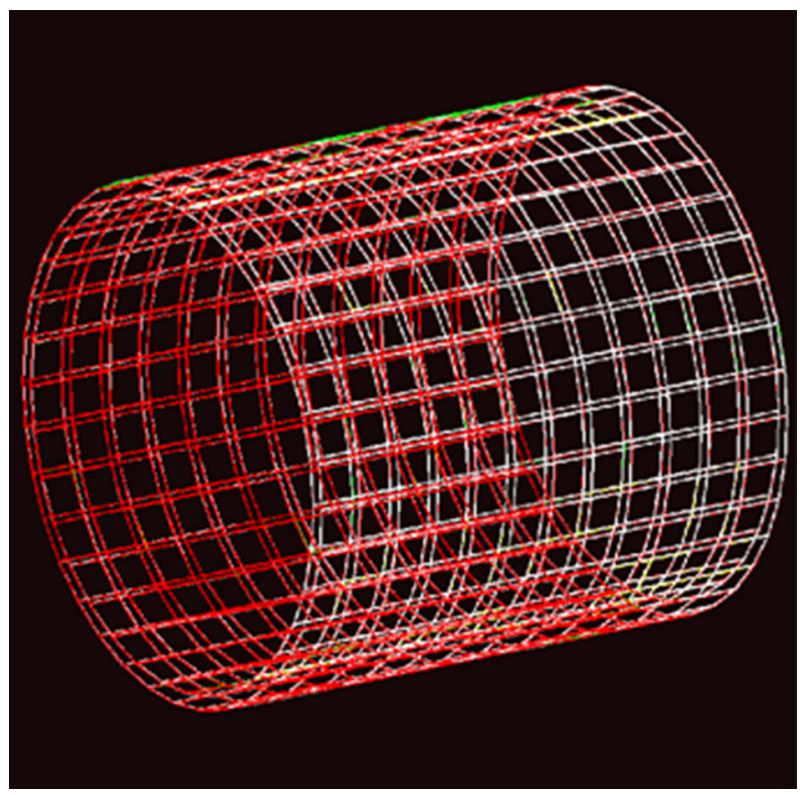

Fig. 3 Schematic drawing of one of the three barrel stations of the IDEA muon detector. The modular structure of the station, based on a large mosaic of same size $\mu$ RWELL detectors is clearly visible

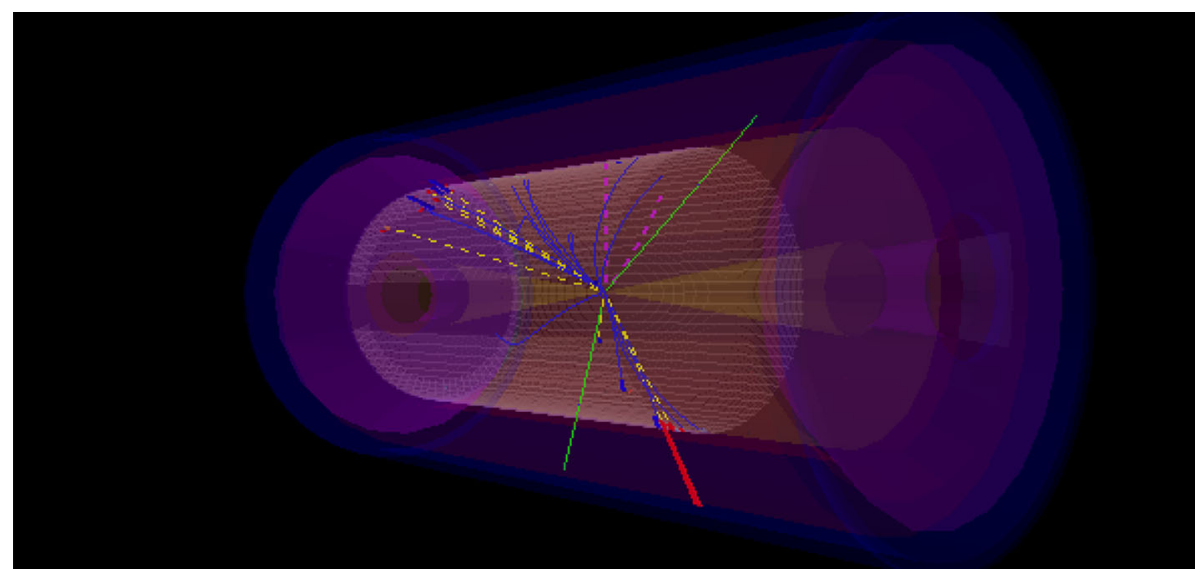

Fig. 4 Display of an $\mathrm{e}^{+} \mathrm{e}^{-} \rightarrow \mathrm{HZ}$, with $\mathrm{H} \rightarrow$ anything, $\mathrm{Z} \rightarrow \mu^{+} \mu^{-}$simulated event with the IDEA detector. The two muon tracks are displayed in green 
Funding This project is co-funded from the European Union's Horizon 2020 research and innovation programme under grant agreement No 95175 .

Open Access This article is licensed under a Creative Commons Attribution 4.0 International License, which permits use, sharing, adaptation, distribution and reproduction in any medium or format, as long as you give appropriate credit to the original author(s) and the source, provide a link to the Creative Commons licence, and indicate if changes were made. The images or other third party material in this article are included in the article's Creative Commons licence, unless indicated otherwise in a credit line to the material. If material is not included in the article's Creative Commons licence and your intended use is not permitted by statutory regulation or exceeds the permitted use, you will need to obtain permission directly from the copyright holder. To view a copy of this licence, visit http://creativecommons.org/licenses/by/4.0/.

\section{References}

1. FCC collaboration, A. Abada et al., FCC Physics Opportunities: Future Circular Collider Conceptual Design Report Volume 1. Eur. Phys. J. C 79, 474 (2019)

2. T. Hebbeker, K. Hoepfner, Muon Spectrometers, in Handbook of Particle Detection and Imaging. ed. by C. Grupen, I. Buvat (Springer, Berlin, Heidelberg, 2012), pp. 473-496

3. D. Renker, Geiger mode avalanche photodiodes, history, properties and problems. Nucl. Instrum. Meth. A 567, 48-56 (2006)

4. D. Renker, E. Lorenz, Advances in solid state photon detectors. JINST 4, P04004 (2009)

5. ATLAS collaboration, G. Aad et al., The ATLAS Experiment at the CERN Large Hadron Collider. JINST 3, S08003 (2008)

6. CMS collaboration, S. Chatrchyan et al., The CMS experiment at the CERN LHC. JINST 3, S08004 (2008)

7. B. Aubert et al., The BABAR detector. Nucl. Instrum. Meth. A 479, 1-116 (2002)

8. G. Ascoli, L. Holloway, I. Karliner, U. Kruse, R. Sard, V. Simaitis et al., CDF central muon detector. Nucl. Instrum. Meth. A 268, 33-40 (1988)

9. G. Charpak, R. Bouclier, T. Bressani, J. Favier, č. Zupančič, The use of multiwire proportional counters to select and localize charged particles. Nuclear Instruments and Methods 62, 262-268 (1968)

10. G. Charpak, F. Sauli, Multiwire proportional chambers and drift chambers. Nucl. Instrum. Methods 162, 405-428 (1979)

11. M. Livan, Monitored drift tubes in ATLAS. Nucl. Instrum. Meth. A 384, 214-218 (1996)

12. F. Bauer, U. Bratzler, H. Dietl, H. Kroha, T. Lagouri, A. Manz et al., Construction and test of MDT chambers for the ATLAS muon spectrometer. Nucl. Instrum. Meth. A 461, 17-20 (2001)

13. G. Battistoni, E. Iarocci, G. Nicoletti, L. Trasatti, Detection of induced pulses in proportional wire devices with resistive cathodes. Nucl. Instrum. Meth. 152, 423 (1978)

14. R. Santonico, R. Cardarelli, Development of Resistive Plate Counters. Nucl. Instrum. Meth. A187, 377 (1981)

15. R. Cardarelli, R. Santonico, Progress in resistive plate chambers. Nucl. Instrum. Meth. A263, 83 (1993)

16. N. Bacchetta, J.J. Blaising, E. Brondolin, M. Dam, D. Dannheim, K. Elsener et al., CLD - A Detector Concept for the FCC-ee, arXiv:1911.12230

17. M. Titov, Perspectives of Micro-Pattern Gaseous Detector Technologies for Future Physics Projects, arXiv: 1308.3047

18. F. Sauli, GEM: A new concept for electron amplification in gas detectors. Nucl. Instrum. Meth. A386, 531 (1997)

19. G. Bencivenni, R. De Oliveira, G. Morello, M. Poli Lener, The micro-Resistive WELL detector: a compact spark-protected single amplification-stage MPGD. JINST 10, P02008 (2015)

20. G. Bencivenni, L. Benussi, L. Borgonovi, R. De Oliveira, P. De Simone, G. Felici et al., The $\mu$-RWELL detector. JINST 114, P0517 (2017)

21. G. Bencivenni, R. De Oliveira, G. Felici, M. Gatta, G. Morello, A. Ochi et al., Performance of $\mu$-RWELL detector versus resistivity of the resistive stage. Nucl. Instrum. Meth. A886, 36 (2018)

22. I. Giomataris et al., Micromegas: a high-granularity position-sensitive gaseous detector for high particleflux environments. Nucl. Instrum. Meth. A376, 29 (1996)

23. C. Altunbas et al., Construction, test and commissioning of the triple-GEM tracking detector for COMPASS. Nucl. Instrum. Meth. A 490, 177-203 (2002)

24. B. Ketzer, J. Ehlers, J. Friedrich, B. Grube, S. Kappler, I. Konorov et al., A fast tracker for COMPASS based on the GEM. Nucl. Phys. B Proc. Suppl. 125, 368-373 (2003) 
25. J. Agarwala, M. Alexeev, C. Azevedo, F. Bradamante, A. Bressan, M. Büchele et al., MPGD-based photon detectors for the upgrade of COMPASS RICH-1 and beyond. JINST 15, C09063-C09063 (2020)

26. M. Bagliesi, M. Berretti, E. Brucken, R. Cecchi, E. David, F. Garcia et al., The TOTEM T2 telescope based on triple-GEM chambers. Nucl. Instrum. Meth. A 617, 134-137 (2010)

27. G. Bencivenni, D. Domenici, An ultra-light cylindrical GEM detector as inner tracker at KLOE-2. Nucl. Instrum. Meth. A 581, 221-224 (2007)

28. T. Kawamoto, S. Vlachos, L. Pontecorvo, J. Dubbert, G. Mikenberg, P. Iengo et al., New Small Wheel Technical Design Report, Tech. Rep. CERN-LHCC-2013-006. ATLAS-TDR-020 (Jun, 2013)

29. M. Iodice, Micromegas detectors for the muon spectrometer upgrade of the ATLAS experiment. JINST 10, C02026-C02026 (2015)

30. A. Colaleo, A. Safonov, A. Sharma, M. Tytgat, M. Akl, M. Abbrescia et al., CMS Technical Design Report for the Muon Endcap GEM Upgrade, Tech. Rep. CERN-LHCC-2015-012. CMS-TDR-013 (Jun, 2015)

31. D. Abbaneo, M. Abbas, M. Abbrescia, A. Abdelalim, M.A. Akl, W. Ahmed et al., Upgrade of the CMS muon system with triple-GEM detectors. JINST 9, C10036 (2014)

32. P. Giacomelli, Muon detectors and Micro-Pattern Gas Detectors. Int. J. Modern Phys. A 34, 1940014 (2019)

33. The CEPC Study Group, CEPC Conceptual Design Report: Volume 2 - Physics Detector, arXiv: 1811.10545

34. M. Poli Lener, G. Bencivenni, G. Felici, M. Gatta, G. Morello, R. De Oliveira, The micro-RWELL detector. PoS MPGD2017, 019 (2019)

35. R. Aly et al., First test-beam results obtained with IDEA, a detector concept designed for future lepton colliders. Nucl. Instrum. Meth. A 958, 162088 (2020) 